\title{
Norms and Spread of the Fibonacci and Lucas RSFMLR Circulant Matrices
}

\author{
Wenai Xu and Zhaolin Jiang \\ Department of Mathematics, Linyi University, Linyi, Shandong 276000, China \\ Correspondence should be addressed to Zhaolin Jiang; jzh1208@sina.com
}

Received 25 July 2014; Accepted 16 September 2014

Academic Editor: Zidong Wang

Copyright (C) 2015 W. Xu and Z. Jiang. This is an open access article distributed under the Creative Commons Attribution License, which permits unrestricted use, distribution, and reproduction in any medium, provided the original work is properly cited.

Circulant type matrices have played an important role in networks engineering. In this paper, firstly, some bounds for the norms and spread of Fibonacci row skew first-minus-last right (RSFMLR) circulant matrices and Lucas row skew first-minus-last right (RSFMLR) circulant matrices are given. Furthermore, the spectral norm of Hadamard product of a Fibonacci RSFMLR circulant matrix and a Lucas RSFMLR circulant matrix is obtained. Finally, the Frobenius norm of Kronecker product of a Fibonacci RSFMLR circulant matrix and a Lucas RSFMLR circulant matrix is presented.

\section{Introduction}

Circulant type matrices have been put on the firm basis with the work in [1-4] and so on. Circulant type matrices have significant applications in networks systems. In [5], some preliminary results on the dynamical behaviours of some specific nonmonotone Boolean automata networks which are called xor circulant networks were showed. In [6], the authors proposed a special class of the feedback delay network using circulant matrices. In [7], the impact of interior symmetries on the multiplicity of the eigenvalues of the Jacobian matrix at a fully synchronous equilibrium for the coupled cell systems associated with homogeneous networks was analyzed by Aguiar and Ruan, which was based on the circulant adjacency matrices of the networks induced by these interior symmetries. Exploiting the circulant structure of the channel matrices, the realistic near fast fading scenarios with circulant frequency selective channels were analysed by Eghbali et al. in [8]. The existence of doubly periodic travelling waves in cellular networks involving the discontinuous Heaviside step function by circulant matrix was studied by Wang and Cheng in [9].

The Fibonacci and Lucas sequences $F_{n}$ and $L_{n}$ are defined by the recurrence relations $[10,11]$ :

$$
\begin{gathered}
F_{0}=0, \quad F_{1}=1, \quad F_{n}=F_{n-1}+F_{n-2} \quad \text { for } n \geq 2 \\
L_{0}=2, \quad L_{1}=1, \quad L_{n}=L_{n-1}+L_{n-2} \quad \text { for } n \geq 2
\end{gathered}
$$

If we start from $n=0$, then Fibonacci and Lucas sequences are given by

$$
\begin{array}{cccccccccc}
n & 0 & 1 & 2 & 3 & 4 & 5 & 6 & 7 & \cdots \\
F_{n} & 0 & 1 & 1 & 2 & 3 & 5 & 8 & 13 & \cdots \\
L_{n} & 2 & 1 & 3 & 4 & 7 & 11 & 18 & 29 & \cdots
\end{array}
$$

In [10], their Binet forms are given by

$$
\begin{aligned}
& F_{n}=\frac{1}{\sqrt{5}}\left[\left(\frac{1+\sqrt{5}}{2}\right)^{n}-\cos (\pi n)\left(\frac{1+\sqrt{5}}{2}\right)^{-n}\right], \\
& L_{n}=\left(\frac{1+\sqrt{5}}{2}\right)^{n}+\cos (\pi n)\left(\frac{1+\sqrt{5}}{2}\right)^{-n} .
\end{aligned}
$$

The following sum formulations for the Fibonacci and Lucas numbers are well known [11]:

$$
\begin{gathered}
\sum_{s=1}^{n-1} F_{s}^{2}=F_{n} F_{n-1}, \\
\sum_{s=1}^{n-1} L_{s}^{2}=L_{n} L_{n-1}-2, \\
\sum_{s=1}^{n} F_{s} F_{s-1}= \begin{cases}F_{n}^{2}, & n \text { even, } \\
F_{n}^{2}-1, & n \text { odd, }\end{cases} \\
\sum_{s=1}^{n} L_{s} L_{s-1}= \begin{cases}L_{n}^{2}-4, & n \text { even, } \\
L_{n}^{2}+1, & n \text { odd. }\end{cases}
\end{gathered}
$$


Lately, some authors studied the problems of the norms of some special matrices [11-21]. The author [11] found upper and lower bounds for the spectral norms of Toeplitz matrices such that $a_{i j} \equiv F_{i-j}$ and $b_{i-j} \equiv L_{i-j}$. In [13], the authors obtain upper and lower bounds for the spectral norms of matrices $A=C_{r}\left(F_{k, 0}, F_{k, 1}, \ldots, F_{k, n-1}\right)$ and $B=$ $C_{r}\left(L_{k, 0}, L_{k, 1}, \ldots, L_{k, n-1}\right)$, where $\left\{F_{k, n}\right\}_{n \in N}$ and $\left\{L_{k, n}\right\}_{n \in N}$ are $k$ Fibonacci and $k$-Lucas sequences, respectively, and they also give the bounds for the spectral norms of Kronecker and Hadamard products of these special matrices, respectively [14]. Solak and Bozkurt [16] have found out upper and lower bounds for the spectral norms of Cauchy-Toeplitz and Cauchy-Hankel matrices. Solak [18-20] has defined $A=\left[a_{i j}\right]$ and $B=\left[b_{i j}\right]$ as $n \times n$ circulant matrices, where $a_{i j} \equiv$ $F_{(\bmod (j-i, n))}$ and $b_{i j} \equiv L_{(\bmod (j-i, n))}$; then he has given some bounds for the $A$ and $B$ matrices concerned with the spectral and Euclidean norms.

In this paper, we define two kinds of special matrices as follows.

A Fibonacci row skew first-minus-last right (RSFMLR) circulant matrix is defined as a square matrix of the form

$$
\left(\begin{array}{cccc}
F_{0} & F_{1} & \cdots & F_{n-1} \\
-F_{n-1} & F_{0}-F_{n-1} & F_{1} & F_{n-2} \\
\vdots & -F_{n-1}-F_{n-2} & \ddots & \vdots \\
-F_{2} & \ddots & \ddots & F_{1} \\
-F_{1} & -F_{2}-F_{1} & \cdots & F_{0}-F_{n-1}
\end{array}\right) \text {. }
$$

A Lucas row skew first-minus-last right (RSFMLR) circulant matrix is defined as a square matrix of the form

$$
\left(\begin{array}{cccc}
L_{0} & L_{1} & \cdots & L_{n-1} \\
-L_{n-1} & L_{0}-L_{n-1} & L_{1} & L_{n-2} \\
\vdots & -L_{n-1}-L_{n-2} & \ddots & \vdots \\
-L_{2} & \ddots & \ddots & L_{1} \\
-L_{1} & -L_{2}-L_{1} & \cdots & L_{0}-L_{n-1}
\end{array}\right)
$$

Obviously, the RSFMLR circulant matrix is determined by its first row, and RSFMLR circulant matrix is a $x^{n}+x+1$ circulant matrix [22]. that is,

We define $\Theta_{(-1,-1)}$ as the basic RSFMLR circulant matrix;

$$
\begin{aligned}
\Theta_{(-1,-1)} & =\left(\begin{array}{ccccc}
0 & 1 & 0 & \cdots & 0 \\
\vdots & \ddots & \ddots & \ddots & \vdots \\
\vdots & & \ddots & \ddots & 0 \\
0 & \cdots & \cdots & 0 & 1 \\
-1 & -1 & 0 & \cdots & 0
\end{array}\right)_{n \times n} \\
& =\operatorname{RSFMLRcircfr}(0,1,0, \ldots, 0) .
\end{aligned}
$$

It is easily verified that $g(x)=x^{n}+x+1$ has no repeated roots in its splitting field and $g(x)=x^{n}+x+1$ is both the minimal polynomial and the characteristic polynomial of the matrix $\Theta_{(-1,-1)}$. In addition, $\Theta_{(-1,-1)}$ is nonderogatory and satisfies $\Theta_{(-1,-1)}^{j}=\operatorname{RFMLRcircfr}(\underbrace{0, \ldots, 0}_{j}, 1, \underbrace{0, \ldots, 0}_{n-j-1})$ and $\Theta_{(-1,-1)}^{n}=$ $-I_{n}+\Theta_{(-1,-1)}$.
As we all know, letting $A=\operatorname{RSFMLRcircfr}\left(a_{0}, a_{1}, \ldots\right.$, $\left.a_{n-1}\right)$ be a RSFMLR circulant matrix with the first row $\left(a_{0}\right.$, $\left.a_{1}, \ldots, a_{n-1}\right)$, it is clear that

$$
\begin{aligned}
A & =\operatorname{RSFMLRcircfr}\left(a_{0}, a_{1}, \ldots, a_{n-1}\right) \\
& =\sum_{i=0}^{n-1} a_{i} \Theta_{(-1,-1)}^{i} .
\end{aligned}
$$

Thus, $A$ is a RSFMLR circulant matrix if and only if $A=f\left(\Theta_{(-1,-1)}\right)$ for some polynomial $f(x)$. The polynomial $f(x)=\sum_{i=0}^{n-1} a_{i} x^{i}$ will be called the representer of the RSFMLR circulant matrix $A$. By (11), it is clear that $A$ is a RSFMLR circulant matrix if and only if $A$ commutes with $\Theta_{(-1,-1)}$; that is, $A \Theta_{(-1,-1)}=\Theta_{(-1,-1)} A$.

In addition to the algebraic properties that can be easily derived from the representation (11), we mention that RSFMLR circulant matrices have very nice structure. The product of two RSFMLR circulant matrices is a RSFMLR circulant matrix and $A^{-1}$ is a RSFMLR circulant matrix too.

Let $A=\left(a_{i j}\right)$ be an $n \times n$ matrix. The Euclidean (or Frobenius) norm, the spectral norm, the maximum column sum matrix norm, and the maximum row sum matrix norm of the matrix $A$ are, respectively [11],

$$
\begin{aligned}
\|A\|_{F} & =\left(\sum_{i, j=1}^{n}\left|a_{i j}\right|^{2}\right)^{1 / 2}, \\
\|A\|_{2} & =\left(\max _{1 \leq i \leq n} \lambda_{i}\left(A^{*} A\right)\right)^{1 / 2}, \\
\|A\|_{1} & =\max _{1 \leq j \leq n} \sum_{i=1}^{n}\left|a_{i j}\right| \\
\|A\|_{\infty} & =\max _{1 \leq i \leq n} \sum_{j=1}^{n}\left|a_{i j}\right|,
\end{aligned}
$$

where $A^{*}$ denotes the conjugate transpose of $A$. The following inequality holds:

$$
\frac{1}{\sqrt{n}}\|A\|_{F} \leq\|A\|_{2} \leq\|A\|_{F} .
$$

Let $A=\left[a_{i j}\right]$ and $B=\left[b_{i j}\right]$ be $n \times n$ matrices. The Hadamard product of $A$ and $B$ is defined by $A \circ B=\left[a_{i j} b_{i j}\right]$. If $\|\cdot\|$ is any norm on $n \times m$ matrices, then $[18,23]$

$$
\|A \circ B\| \leq\|A\| \cdot\|B\| .
$$

Kronecker product of $A$ and $B$ is given to be [18]

$$
A \otimes B=\left[\begin{array}{ccc}
a_{11} B & \cdots & a_{1 m} B \\
\vdots & & \vdots \\
a_{n 1} B & \cdots & a_{n m} B
\end{array}\right] .
$$

Then [18]

$$
\|A \otimes B\|_{F}=\|A\|_{F}\|B\|_{F} .
$$


Let $A=\left(a_{i j}\right)$ be an $n \times n$ matrix with eigenvalues $\lambda_{i}, i=$ $1,2, \ldots, n$. The spread of $A$ is defined as $[24,25]$

$$
s(A)=\max _{i, j}\left|\lambda_{i}-\lambda_{j}\right| .
$$

An upper bound for the spread due to Mirsky [24] states that

$$
s(A) \leq \sqrt{2\|A\|_{F}^{2}-\frac{2}{n}|\operatorname{tr} A|^{2}},
$$

where $\|A\|_{F}$ denotes the Frobenius norm of $A$ and $\operatorname{tr} A$ is the trace of $A$.

\section{Norms and Spread of Fibonacci RSFMLR Circulant Matrices}

Theorem 1. Let $A=R S F M L R \operatorname{circfr}\left(F_{0}, F_{1}, \ldots, F_{n-1}\right)$ be a Fibonacci RSFMLR circulant matrix, where $\left\{F_{i}\right\}_{0 \leq i \leq n-1}$ denote Fibonacci numbers given by (1); then two kinds of norms of $A$ are given by

$$
\|A\|_{1}=\|A\|_{\infty}=2\left(F_{n+1}-1\right) .
$$

Proof. The matrix $A$ is of the form (8), by (14), (15); then we have

$$
\begin{aligned}
\|A\|_{1} & =\max _{1 \leq j \leq n} \sum_{i=1}^{n}\left|a_{i j}\right| \\
& =\sum_{i=1}^{n-1} F_{i}+F_{n+1}-F_{2}, \\
\|A\|_{\infty} & =\max _{1 \leq i \leq n} \sum_{j=1}^{n}\left|a_{i j}\right| \\
& =\sum_{i=1}^{n-1} F_{i}+F_{n+1}-F_{2} .
\end{aligned}
$$

Since the Fibonacci sequences $F_{n}$ are defined by the recurrence relations (1), then we obtain

$$
F_{n-1}=F_{n}-F_{n-2} \quad n \geq 2 .
$$

To sum up, we can get

$$
\sum_{s=1}^{n-1} F_{s}=F_{n+1}-F_{2} .
$$

Then

$$
\|A\|_{1}=\|A\|_{\infty}=2\left(F_{n+1}-1\right),
$$

which completes the proof.

Theorem 2. Let $A=R S F M L R \operatorname{circfr}\left(F_{0}, F_{1}, \ldots, F_{n-1}\right)$ be a Fibonacci RSFMLR circulant matrix, where $\left\{F_{i}\right\}_{0 \leq i \leq n-1}$ denote Fibonacci numbers given by (1); then

$$
\begin{gathered}
\sqrt{\frac{\Gamma}{n}} \leq\|A\|_{2}, \\
\|A\|_{2} \leq 2\left(F_{n+1}-1\right),
\end{gathered}
$$

where

$$
\begin{aligned}
\Gamma= & F_{1}^{2}+(3 n-4) F_{n-1}^{2}+(n-1) F_{n-3} F_{n-2} \\
& +(2 n-4) F_{n-1} F_{n-2}+(2 n-3) F_{n-2}^{2} .
\end{aligned}
$$

Proof. Since $F_{n+2}=F_{n+1}+F_{n}$ and $F(0)=0$ given by (1), the matrix $A$ is of the form

$$
\left(\begin{array}{cccc}
F_{0} & F_{1} & F_{n-2} & F_{n-1} \\
-F_{n-1} & -F_{n-1} & \cdots & F_{n-2} \\
\vdots & -F_{n-1}-F_{n-2} & \ddots & \vdots \\
-F_{2} & \vdots & \ddots & F_{1} \\
-F_{1} & -F_{3} & -F_{n-1}-F_{n-2} & -F_{n-1}
\end{array}\right)
$$

We know that $(1 / \sqrt{n})\|A\|_{F} \leq\|A\|_{2} \leq\|A\|_{F}$ from equivalent norms. By (5), we can get

$$
\begin{aligned}
\|A\|_{F}^{2}= & n \sum_{i=0}^{n-1} F_{i}^{2}+\sum_{i=1}^{n-1} i F_{i}^{2}+2 \sum_{i=1}^{n-2} i F_{i} F_{i+1} \\
= & n \sum_{i=0}^{n-1} F_{i}^{2}+\sum_{k=1}^{n-1} \sum_{i=n-k}^{n-1} F_{i}^{2}+2 \sum_{k=1}^{n-2} \sum_{i=n-k-1}^{n-2} F_{i} F_{i+1} \\
= & n \sum_{i=0}^{n-1} F_{i}^{2}+\sum_{k=1}^{n-1}\left(\sum_{i=0}^{n-1} F_{i}^{2}-\sum_{i=0}^{n-k-1} F_{i}^{2}\right) \\
& +2 \sum_{k=1}^{n-2}\left(\sum_{i=0}^{n-2} F_{i} F_{i+1}-\sum_{i=0}^{n-k-2} F_{i} F_{i+1}\right) \\
= & F_{1}^{2}+(3 n-4) F_{n-1}^{2}+(n-1) F_{n-3} F_{n-2} \\
& +(2 n-4) F_{n-1} F_{n-2}+(2 n-3) F_{n-2}^{2} .
\end{aligned}
$$

Then

$$
\frac{1}{\sqrt{n}}\|A\|_{F}=\sqrt{\frac{\Gamma}{n}},
$$

where

$$
\begin{aligned}
\Gamma= & F_{1}^{2}+(3 n-4) F_{n-1}^{2}+(n-1) F_{n-3} F_{n-2} \\
& +(2 n-4) F_{n-1} F_{n-2}+(2 n-3) F_{n-2}^{2} .
\end{aligned}
$$

We have 
On the other hand, suppose that

$$
\begin{aligned}
M_{1}= & \left(\begin{array}{cccccc}
0 & 1 & 0 & \cdots & 0 & 0 \\
0 & 0 & 1 & \ddots & 0 & 0 \\
0 & 0 & 0 & \ddots & 0 & 0 \\
\vdots & \vdots & \vdots & \ddots & \ddots & \vdots \\
0 & 0 & 0 & \cdots & 0 & 1 \\
-1 & 0 & 0 & \cdots & 0 & 0
\end{array}\right), \\
M_{2}= & \left(\begin{array}{cccccc}
0 & 0 & 0 & \cdots & 0 & 0 \\
0 & 0 & 0 & \cdots & 0 & 0 \\
0 & 1 & 0 & \cdots & 0 & 0 \\
\vdots & \ddots & \ddots & \ddots & \vdots & \vdots \\
0 & 0 & 0 & \ddots & 0 & 0 \\
0 & 0 & 0 & \cdots & 1 & 0
\end{array}\right), \\
M_{3}= & \left(\begin{array}{cccccc}
0 & 0 & 0 & \cdots & 0 & 0 \\
0 & -1 & 0 & \cdots & 0 & 0 \\
0 & 0 & -1 & \cdots & 0 & 0 \\
\vdots & \vdots & \vdots & \ddots & \vdots & \vdots \\
0 & 0 & 0 & \cdots & -1 & 0 \\
0 & 0 & 0 & \cdots & 0 & -1
\end{array}\right) .
\end{aligned}
$$

Then

$$
A=\sum_{i=0}^{n-1} F_{i} M_{1}^{i}-\sum_{i=1}^{n-2} F_{n-i-1} M_{2}^{i}+F_{n-1} M_{3} .
$$

We can get

$$
\begin{aligned}
\|A\|_{2} & =\left\|\sum_{i=0}^{n-1} F_{i} M_{2}^{i}+\sum_{i=1}^{n-2} F_{n-i-1} M_{2}^{i}+F_{n-1} M_{3}\right\|_{2} \\
& \leq \sum_{i=0}^{n-1} F_{i}\left\|M_{1}\right\|_{2}^{i}-\sum_{i=1}^{n-2} F_{n-i-1}\left\|M_{2}\right\|_{2}^{i}+F_{n-1}\left\|M_{3}\right\|_{2} .
\end{aligned}
$$

Furthermore,

$$
\begin{aligned}
& M_{1}^{H} M_{1}=\left(\begin{array}{cccc}
1 & 0 & \cdots & 0 \\
0 & 1 & \cdots & 0 \\
\vdots & \vdots & \ddots & \vdots \\
0 & 0 & \cdots & 1
\end{array}\right) \\
& M_{2}^{H} M_{2}=\left(\begin{array}{ccccc}
0 & 0 & \cdots & 0 & 0 \\
0 & 1 & \cdots & 0 & 0 \\
\vdots & \vdots & \ddots & \vdots & \vdots \\
0 & 0 & \cdots & 1 & 0 \\
0 & 0 & \cdots & 0 & 0
\end{array}\right) \text {, } \\
& M_{3}^{H} M_{3}=\left(\begin{array}{cccc}
0 & 0 & \cdots & 0 \\
0 & 1 & \cdots & 0 \\
\vdots & \vdots & \ddots & \vdots \\
0 & 0 & \cdots & 1
\end{array}\right) \text {. }
\end{aligned}
$$

We obtain

$$
\left\|M_{1}\right\|_{2}=\left\|M_{2}\right\|_{2}=\left\|M_{3}\right\|_{2}=1 \text {. }
$$

The other result is obtained as follows:

$$
\begin{aligned}
\|A\|_{2} & \leq \sum_{i=1}^{n-1} F_{i}\left\|M_{1}\right\|_{2}^{i}+\sum_{i=1}^{n-2} F_{n-i-1}\left\|M_{2}\right\|_{2}^{i}+F_{n-1}\left\|M_{3}\right\|_{2} \\
& =2 \sum_{i=0}^{n-1} F_{i}=2\left(F_{n+1}-1\right),
\end{aligned}
$$

which completes the proof.

Theorem 3. Let $A=R S F M L R \operatorname{circfr}\left(F_{0}, F_{1}, \ldots, F_{n-1}\right)$ be a Fibonacci RSFMLR circulant matrix, where $\left\{F_{i}\right\}_{0 \leq i \leq n-1}$ denote Fibonacci numbers given by (1); then the bound for the spread of $A$ is

$$
s(A) \leq \sqrt{\tau_{1}(n)-\frac{2}{n} \tau_{2}(n)}
$$

where

$$
\begin{aligned}
& \tau_{1}(n)=2\left(F_{1}^{2}+(2 n-1) F_{n-1}^{2}+(n-1) F_{n-3} F_{n-2}\right. \\
& \left.+(2 n-4) F_{n-1} F_{n-2}+(2 n-3) F_{n-2}^{2}\right), \\
& \tau_{2}(n)=\left[(n-1) F_{n-1}\right]^{2} .
\end{aligned}
$$

Proof. The trace of $A$ is $\operatorname{tr} A=n F_{0}+(n-1) F_{n-1}$. By Theorem 2 and inequation (21), we have

$$
s(A) \leq \sqrt{2\|A\|_{F}^{2}-\frac{2}{n} \operatorname{tr} A^{2}},
$$

where

$$
\begin{aligned}
\|A\|_{F}^{2}= & F_{1}^{2}+(3 n-4) F_{n-1}^{2}+(n-1) F_{n-3} F_{n-2} \\
+ & (2 n-4) F_{n-1} F_{n-2}+(2 n-3) F_{n-2}^{2} \\
& \operatorname{tr} A=n F_{0}-(n-1) F_{n-1} .
\end{aligned}
$$

We can get

$$
s(A) \leq \sqrt{\tau_{1}(n)-\frac{2}{n} \tau_{2}(n)},
$$

where

$$
\begin{aligned}
& \tau_{1}(n)=2\left(F_{1}^{2}+(2 n-1) F_{n-1}^{2}+(n-1) F_{n-3} F_{n-2}\right. \\
& \left.\quad+(2 n-4) F_{n-1} F_{n-2}+(2 n-3) F_{n-2}^{2}\right), \\
& \tau_{2}(n)=\left[(n-1) F_{n-1}\right]^{2},
\end{aligned}
$$

which completes the proof.

\section{Norms and Spread of Lucas RSFMLR Circulant Matrices}

Theorem 4. Let $B=R S F M L R \operatorname{circfr}\left(L_{0}, L_{1}, \ldots, L_{n-1}\right)$ be a Lucas RSFMLR circulant matrix, where $\left\{L_{i}\right\}_{0 \leq i \leq n-1}$ denote Lucas numbers given by (2); then two kinds of norms of $B$ are given by

$$
\|B\|_{1}=\|B\|_{\infty}=2\left(L_{n+1}-3\right)+2 .
$$


Proof. The matrix $B$ is of the form (9), by (14), (15); then we get

$$
\begin{gathered}
\|B\|_{1}=\max _{1 \leq j \leq n} \sum_{i=1}^{n}\left|b_{i j}\right|=\sum_{i=1}^{n-1} L_{i}+L_{0}+L_{n+1}-L_{2}, \\
\|B\|_{\infty}=\max _{1 \leq i \leq n} \sum_{j=1}^{n}\left|b_{i j}\right|=\sum_{i=1}^{n-1} L_{i}+L_{0}+L_{n+1}-L_{2} .
\end{gathered}
$$

Since the Lucas sequences $L_{n}$ are defined by the recurrence relations (2), then we obtain

$$
L_{n-1}=L_{n}-L_{n-2} \quad n \geq 2 .
$$

To sum up, we can get

$$
\sum_{s=1}^{n-1} L_{s}=L_{n+1}-L_{2}
$$

Then

$$
\|B\|_{1}=\|B\|_{\infty}=2\left(L_{n+1}-3\right)+2,
$$

which completes the proof.

Theorem 5. Let $B=R S F M L R \operatorname{Rircfr}\left(L_{0}, L_{1}, \ldots, L_{n-1}\right)$ be a Lucas RSFMLR circulant matrix, where $\left\{L_{i}\right\}_{0 \leq i \leq n-1}$ denote Lucas numbers given by (2); then

$$
\begin{gathered}
\sqrt{\frac{\Pi}{n}} \leq\|B\|_{2}, \\
\|B\|_{2} \leq 2\left(L_{n+1}-2\right),
\end{gathered}
$$

where

$$
\begin{aligned}
\Pi= & L_{1}^{2}+(3 n-4) L_{n-1}^{2}+(n-1) L_{n-3} L_{n-2} \\
& +(2 n-3) L_{n-2}^{2}-(4 n-4) L_{n-1} \\
& +(2 n-4) L_{n-1} L_{n-2}+2 n+2 .
\end{aligned}
$$

Proof. Since $L_{n+2}=L_{n+1}+L_{n}$ and $L(0)=2$, the matrix $B$ is of the form

$$
\left(\begin{array}{cccc}
L_{0} & L_{1} & L_{n-2} & L_{n-1} \\
-L_{n-1} & L_{0}-L_{n-1} & \cdots & L_{n-2} \\
\vdots & -L_{n-1}-L_{n-2} & \ddots & \vdots \\
-L_{2} & \vdots & \ddots & L_{1} \\
-L_{1} & -L_{3} & -L_{n-1}-L_{n-2} & L_{0}-L_{n-1}
\end{array}\right)
$$

We know that $(1 / \sqrt{n})\|B\|_{F} \leq\|B\|_{2} \leq\|B\|_{F}$ from equivalent norms. By (6), we can get

$$
\begin{aligned}
\|B\|_{F}^{2}= & n \sum_{i=0}^{n-1} L_{i}^{2}+\sum_{i=1}^{n-1} i L_{i}^{2}+2 \sum_{i=1}^{n-2} i L_{i} L_{i+1}-4(n-1) L_{n-1} \\
= & n \sum_{i=0}^{n-1} L_{i}^{2}+\sum_{k=1}^{n-1} \sum_{i=n-k}^{n-1} L_{i}^{2}+2 \sum_{k=1}^{n-2} \sum_{i=n-k-1}^{n-2} L_{i} L_{i+1} \\
& -4(n-1) L_{n-1}
\end{aligned}
$$

$$
\begin{aligned}
= & n \sum_{i=0}^{n-1} L_{i}^{2}+\sum_{k=1}^{n-1}\left(\sum_{i=0}^{n-1} L_{i}^{2}-\sum_{i=0}^{n-k-1} L_{i}^{2}\right) \\
& +2 \sum_{k=1}^{n-2}\left(\sum_{i=0}^{n-2} L_{i} L_{i+1}-\sum_{i=0}^{n-k-2} L_{i} L_{i+1}\right) \\
& -4(n-1) L_{n-1} \\
= & L_{1}^{2}+(3 n-4) L_{n-1}^{2}+(n-1) L_{n-3} L_{n-2} \\
& +(2 n-3) L_{n-2}^{2}-(4 n-4) L_{n-1} \\
& +(2 n-4) L_{n-1} L_{n-2}+2 n+2 .
\end{aligned}
$$

Then

$$
\frac{1}{\sqrt{n}}\|B\|_{F}=\sqrt{\frac{\Pi}{n}} .
$$

We have

$$
\sqrt{\frac{\bar{\Pi}}{n}} \leq\|B\|_{2},
$$

where

$$
\begin{aligned}
\Pi= & L_{1}^{2}+(3 n-4) L_{n-1}^{2}+(n-1) L_{n-3} L_{n-2} \\
& +(2 n-3) L_{n-2}^{2}-(4 n-4) L_{n-1} \\
& +(2 n-4) L_{n-1} L_{n-2}+2 n+2 .
\end{aligned}
$$

On the other hand, supposing that

$$
\begin{aligned}
M_{1}= & \left(\begin{array}{cccccc}
0 & 1 & 0 & \cdots & 0 & 0 \\
0 & 0 & 1 & \ddots & 0 & 0 \\
0 & 0 & 0 & \ddots & 0 & 0 \\
\vdots & \vdots & \vdots & \ddots & \ddots & \vdots \\
0 & 0 & 0 & \cdots & 0 & 1 \\
-1 & 0 & 0 & \cdots & 0 & 0
\end{array}\right) \\
M_{2}= & \left(\begin{array}{cccccc}
0 & 0 & 0 & \cdots & 0 & 0 \\
0 & 0 & 0 & \cdots & 0 & 0 \\
0 & 1 & 0 & \cdots & 0 & 0 \\
\vdots & \ddots & \ddots & \ddots & \vdots & \vdots \\
0 & 0 & 0 & \ddots & 0 & 0 \\
0 & 0 & 0 & \cdots & 1 & 0
\end{array}\right) \\
M_{3}= & \left(\begin{array}{cccccc}
0 & 0 & 0 & \cdots & 0 & 0 \\
0 & -1 & 0 & \cdots & 0 & 0 \\
0 & 0 & -1 & \cdots & 0 & 0 \\
\vdots & \vdots & \vdots & \ddots & \vdots & \vdots \\
0 & 0 & 0 & \cdots & -1 & 0 \\
0 & 0 & 0 & \cdots & 0 & -1
\end{array}\right)
\end{aligned}
$$

then

$$
B=\sum_{i=0}^{n-1} L_{i} M_{1}^{i}-\sum_{i=1}^{n-2} L_{n-i-1} M_{2}^{i}+L_{n-1} M_{3}
$$


We obtain

$$
\begin{aligned}
\|B\|_{2} & =\left\|\sum_{i=0}^{n-1} L_{i} M_{2}^{i}+\sum_{i=1}^{n-2} L_{n-i-1} M_{2}^{i}+L_{n-1} M_{3}\right\|_{2} \\
& \leq \sum_{i=0}^{n-1} L_{i}\left\|M_{1}\right\|_{2}^{i}-\sum_{i=1}^{n-2} L_{n-i-1}\left\|M_{2}\right\|_{2}^{i}+L_{n-1}\left\|M_{3}\right\|_{2} .
\end{aligned}
$$

We have

$$
\begin{aligned}
M_{1}^{H} M_{1} & =\left(\begin{array}{cccc}
1 & 0 & \cdots & 0 \\
0 & 1 & \cdots & 0 \\
\vdots & \vdots & \ddots & \vdots \\
0 & 0 & \cdots & 1
\end{array}\right) \\
M_{2}^{H} M_{2} & =\left(\begin{array}{ccccc}
0 & 0 & \cdots & 0 & 0 \\
0 & 1 & \cdots & 0 & 0 \\
\vdots & \vdots & \ddots & \vdots & \vdots \\
0 & 0 & \cdots & 1 & 0 \\
0 & 0 & \cdots & 0 & 0
\end{array}\right) \\
M_{3}^{H} M_{3} & =\left(\begin{array}{cccc}
0 & 0 & \cdots & 0 \\
0 & 1 & \cdots & 0 \\
\vdots & \vdots & \ddots & \vdots \\
0 & 0 & \cdots & 1
\end{array}\right)
\end{aligned}
$$

We get

$$
\left\|M_{1}\right\|_{2}=\left\|M_{2}\right\|_{2}=\left\|M_{3}\right\|_{2}=1 \text {. }
$$

The other result is obtained as follows:

$$
\begin{aligned}
\|B\|_{2} & \leq \sum_{i=1}^{n-1} L_{i}\left\|M_{1}\right\|_{2}^{i}+\sum_{i=1}^{n-2} L_{n-i-1}\left\|M_{2}\right\|_{2}^{i}+L_{n-1}\left\|M_{3}\right\|_{2} \\
& =2 \sum_{i=0}^{n-1} L_{i}=2\left(L_{n+1}-2\right),
\end{aligned}
$$

which completes the proof.

Theorem 6. Let $B=R S F M L R \operatorname{circfr}\left(L_{0}, L_{1}, \ldots, L_{n-1}\right)$ be a Lucas RSFMLR circulant matrix, where $\left\{L_{i}\right\}_{0 \leq i \leq n-1}$ denote Lucas numbers given by (2); then

$$
s(B) \leq \sqrt{\kappa_{1}(n)-\frac{2}{n} \kappa_{2}(n)},
$$

where

$$
\begin{gathered}
\kappa_{1}(n)=2\left(L_{1}^{2}+(3 n-4) L_{n-1}^{2}+(n-1) L_{n-3} L_{n-2}\right. \\
+(2 n-3) L_{n-2}^{2}-(4 n-4) L_{n-1} \\
\left.+(2 n-4) L_{n-1} L_{n-2}+2 n+2\right), \\
\kappa_{2}(n)=\left[n L_{0}-(n-1) L_{n-1}\right]^{2} .
\end{gathered}
$$

Proof. The trace of $B$ is $\operatorname{tr} B=n L_{0}+(n-1) L_{n-1}$. By Theorem 5 and by inequation (21), we have

$$
s(B) \leq \sqrt{2\|B\|_{F}^{2}-\frac{2}{n} \operatorname{tr} B^{2}},
$$

where

$$
\begin{aligned}
\|B\|_{F}^{2}= & L_{1}^{2}+(3 n-4) L_{n-1}^{2}+(n-1) L_{n-3} L_{n-2} \\
& +(2 n-3) L_{n-2}^{2}-(4 n-4) L_{n-1} \\
& +(2 n-4) L_{n-1} L_{n-2}+2 n+2 \\
& \operatorname{tr} B=n L_{0}-(n-1) L_{n-1} .
\end{aligned}
$$

We obtain

$$
s(B) \leq \sqrt{\kappa_{1}(n)-\frac{2}{n} \kappa_{2}(n)},
$$

where

$$
\begin{aligned}
& \kappa_{1}(n)=2\left(L_{1}^{2}+(3 n-4) L_{n-1}^{2}+(n-1) L_{n-3} L_{n-2}\right. \\
& +(2 n-3) L_{n-2}^{2}-(4 n-4) L_{n-1} \\
& \left.+(2 n-4) L_{n-1} L_{n-2}+2 n+2\right) \\
& \kappa_{2}(n)=\left[n L_{0}-(n-1) L_{n-1}\right]^{2}
\end{aligned}
$$

which completes the proof.

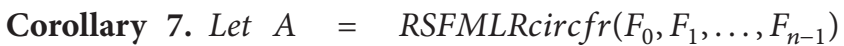
be a Fibonacci RSFMLR circulant matrix and let $B=$ RSFMLRcircfr $\left(L_{0}, L_{1}, \ldots, L_{n-1}\right)$ be a Lucas RSFMLR circulant matrix, where $\left\{F_{i}\right\}_{0 \leq i \leq n-1}$ and $\left\{L_{i}\right\}_{0 \leq i \leq n-1}$ denote Fibonacci numbers and Lucas numbers, respectively; then the spectral norm of Hadamard product of $A$ and $B$ satisfies the following inequality:

$$
\|A \circ B\|_{2} \leq 4\left(F_{n+1}-1\right) \times\left(L_{n+1}-2\right) .
$$

Proof. The proof is trivial by Theorems 2 and 5; we obtain

$$
\|A\|_{2} \leq 2\left(F_{n+1}-1\right), \quad\|B\|_{2} \leq 2\left(L_{n+1}-2\right) .
$$

By inequation (17), we have

$$
\|A \circ B\|_{2} \leq 4\left(F_{n+1}-1\right) \times\left(L_{n+1}-2\right),
$$

which completes the proof.

Corollary 8. Let $A=\operatorname{RSFMLR\operatorname {circfr}}\left(F_{0}, F_{1}, \ldots, F_{n-1}\right)$ be a Fibonacci RSFMLR circulant matrix and let $B=$ $R S F M L R \operatorname{circfr}\left(L_{0}, L_{1}, \ldots, L_{n-1}\right)$ be a Lucas RSFMLR circulant matrix, where $\left\{F_{i}\right\}_{0 \leq i \leq n-1}$ and $\left\{L_{i}\right\}_{0 \leq i \leq n-1}$ denote Fibonacci numbers and Lucas numbers, respectively; then the Frobenius norm of Kronecker product of $A$ and $B$ is

$$
\|A \otimes B\|_{F}=\sqrt{\Gamma} \times \sqrt{\Pi},
$$

where

$$
\begin{aligned}
\Gamma= & F_{1}^{2}+(3 n-4) F_{n-1}^{2}+(n-1) F_{n-3} F_{n-2} \\
& +(2 n-4) F_{n-1} F_{n-2}+(2 n-3) F_{n-2}^{2}, \\
\Pi= & L_{1}^{2}+(3 n-4) L_{n-1}^{2}+(n-1) L_{n-3} L_{n-2} \\
& +(2 n-3) L_{n-2}^{2}-(4 n-4) L_{n-1} \\
& +(2 n-4) L_{n-1} L_{n-2}+2 n+2 .
\end{aligned}
$$


Proof. Since the proof is trivial by Theorems 2 and 5, we obtain

$$
\begin{aligned}
\|A\|_{F}^{2}= & F_{1}^{2}+(3 n-4) F_{n-1}^{2}+(n-1) F_{n-3} F_{n-2} \\
& +(2 n-4) F_{n-1} F_{n-2}+(2 n-3) F_{n-2}^{2}, \\
\|B\|_{F}^{2}= & L_{1}^{2}+(3 n-4) L_{n-1}^{2}+(n-1) L_{n-3} L_{n-2} \\
& +(2 n-3) L_{n-2}^{2}-(4 n-4) L_{n-1} \\
& +(2 n-4) L_{n-1} L_{n-2}+2 n+2 .
\end{aligned}
$$

By (19), then

$$
\|A \otimes B\|_{F}=\sqrt{\Gamma} \times \sqrt{\Pi}
$$

where

$$
\begin{aligned}
\Gamma= & F_{1}^{2}+(3 n-4) F_{n-1}^{2}+(n-1) F_{n-3} F_{n-2} \\
& +(2 n-4) F_{n-1} F_{n-2}+(2 n-3) F_{n-2}^{2}, \\
\Pi= & L_{1}^{2}+(3 n-4) L_{n-1}^{2}+(n-1) L_{n-3} L_{n-2} \\
& +(2 n-3) L_{n-2}^{2}-(4 n-4) L_{n-1} \\
& +(2 n-4) L_{n-1} L_{n-2}+2 n+2,
\end{aligned}
$$

which completes the proof.

\section{Conclusion}

In this study, we define matrices of the following forms: let $A=\operatorname{RSFMLRcircfr}\left(F_{0}, F_{1}, \ldots, F_{n-1}\right)$ be a Fibonacci RSFMLR circulant matrix and let $B=\operatorname{RSFMLRcircfr}\left(L_{0}, L_{1}, \ldots, L_{n-1}\right)$ be a Lucas RSFMLR circulant matrix. Firstly, we get lower and upper bounds for the spectral norms of these matrices. Upper bounds for the spread of the matrix $A$ and the matrix $B$ are given. Afterwards, we obtain some corollaries related to norms of Hadamard and Kronecker products of these matrices. Based on the existing problems in [26-28], we will explore solving these problems by circulant matrices technology.

\section{Conflict of Interests}

The authors declare that there is no conflict of interests regarding the publication of this paper.

\section{Acknowledgments}

The research is supported by the Development Project of Science \& Technology of Shandong Province (Grant no. 2012GGX10115) and the AMEP of Linyi University, China.

\section{References}

[1] P. J. Davis, Circulant Matrices, John Wiley \& Sons, New York, NY, USA, 1979.
[2] Z. L. Jiang and Z. X. Zhou, Circulant Matrices, Chengdu Technology University Publishing Company, Chengdu, China, 1999.

[3] Z. Jiang, "On the minimal polynomials and the inverses of multilevel scaled factor circulant matrices," Abstract and Applied Analysis, vol. 2014, Article ID 521643, 10 pages, 2014.

[4] X. Jiang and K. Hong, "Exact determinants of some special circulant matrices involving four kinds of famous numbers," Abstract and Applied Analysis, vol. 2014, Article ID 273680, 12 pages, 2014.

[5] M. Noual, D. Regnault, and S. Sené, "About non-monotony in Boolean automata networks," Theoretical Computer Science, vol. 504, pp. 12-25, 2013.

[6] D. Rocchesso and J. O. Smith, "Circulant and elliptic feedback delay networks for artificial reverberation," IEEE Transactions on Speech and Audio Processing, vol. 5, no. 1, pp. 51-63, 1997.

[7] M. A. Aguiar and H. Ruan, "Interior symmetries and multiple eigenvalues for homogeneous networks," SIAM Journal on Applied Dynamical Systems, vol. 11, no. 4, pp. 1231-1269, 2012.

[8] H. Eghbali, S. Muhaidat, S. A. Hejazi, and Y. Ding, "Relay selection strategies for single-carrier frequency-domain equalization multi-relay cooperative networks," IEEE Transactions on Wireless Communications, vol. 12, no. 5, pp. 2034-2045, 2013.

[9] G.-Q. Wang and S. S. Cheng, "6-periodic travelling waves in an artificial neural network with bang-bang control," Journal of Difference Equations and Applications, vol. 18, no. 2, pp. 261-304, 2012.

[10] E. G. Kocer, N. Tuglu, and A. Stakhov, "On the $m$-extension of the Fibonacci and Lucas p-numbers," Chaos, Solitons and Fractals, vol. 40, no. 4, pp. 1890-1906, 2009.

[11] M. Akbulak and D. Bozkurt, "On the norms of Toeplitz matrices involving Fibonacci and Lucas numbers," Hacettepe Journal of Mathematics and Statistics, vol. 37, no. 2, pp. 89-95, 2008.

[12] R. Mathias, "The spectral norm of a nonnegative matrix," Linear Algebra and Its Applications, vol. 139, pp. 269-284, 1990.

[13] S. Q. Shen and J. M. Cen, "On the spectral norms of rcirculant matrices with the k-fibonacci and k-lucas numbers," International Journal of Contemporary Mathematical Sciences, vol. 5, no. 12, pp. 569-578, 2010.

[14] S. Q. Shen and J. M. Cen, "On the norms of circulant matrices with the $(\mathrm{k}, \mathrm{h})$-fibonacci and $(\mathrm{k}, \mathrm{h})$-lucas numbers," International Journal of Contemporary Mathematical Sciences, vol. 6, no. 18 , pp. 887-894, 2011.

[15] S. Solak and D. Bozkurt, "Some bounds on $l_{p}$ matrix and $l_{p}$ operator norms of almost circulant, CAUchy-Toeplitz and CAUchyHankel matrices," Mathematical \& Computational Applications, vol. 7, no. 3, pp. 211-218, 2002.

[16] S. Solak and D. Bozkurt, "On the spectral norms of CauchyToeplitz and Cauchy-Hankel matrices," Applied Mathematics and Computation, vol. 140, no. 2-3, pp. 231-238, 2003.

[17] S. Solak and D. Bozkurt, "A note on bound for norms of CauchyHankel matrices," Numerical Linear Algebra with Applications, vol. 10, no. 4, pp. 377-382, 2003.

[18] S. Solak, "On the norms of circulant matrices with the Fibonacci and Lucas numbers," Applied Mathematics and Computation, vol. 160, no. 1, pp. 125-132, 2005.

[19] S. Solak, "Erratum to "on the norms of circulant matrices with the Fibonacci and Lucas numbers"," Applied Mathematics and Computation, vol. 160, no. 1, pp. 125-132, 2005.

[20] S. Solak, "Erratum to "On the norms of circulant matrices with the Fibonacci and Lucas numbers" [Appl. Math. Comput. 160 
(2005) 125-132]," Applied Mathematics and Computation, vol. 190, no. 2, pp. 1855-1856, 2007.

[21] A. Yalc'iner, "Spectral norms of some special circulant matrices," International Journal of Contemporary Mathematical Sciences, vol. 3, no. 35, pp. 1733-1738, 2008.

[22] D. Chillag, "Regular representations of semisimple algebras, separable field extensions, group characters, generalized circulants, and generalized cyclic codes," Linear Algebra and Its Applications, vol. 218, pp. 147-183, 1995.

[23] G. Visick, "A quantitative version of the observation that the Hadamard product is a principal submatrix of the Kronecker product," Linear Algebra and Its Applications, vol. 304, no. 1-3, pp. $45-68,2000$.

[24] L. Mirsky, "The spread of a matrix," Mathematika, vol. 3, pp. 127130, 1956.

[25] R. Sharma and R. Kumar, "Remark on upper bounds for the spread of a matrix," Linear Algebra and its Applications, vol. 438, no. 11, pp. 4359-4362, 2013.

[26] J. Hu, Z. Wang, and H. Gao, "Recursive filtering with random parameter matrices, multiple fading measurements and correlated noises," Automatica, vol. 49, no. 11, pp. 3440-3448, 2013.

[27] D. Ding, Z. Wang, B. Shen, and H. Shu, "State-saturated $H_{\infty}$ filtering with randomly occurring nonlinearities and packet dropouts: the finite-horizon case," International Journal of Robust and Nonlinear Control, vol. 23, no. 16, pp. 1803-1821, 2013.

[28] B. Shen, Z. Wang, D. Ding, and H. Shu, " $H_{\infty}$ state estimation for complex networks with uncertain inner coupling and incomplete measurements," IEEE Transactions on Neural Networks and Learning Systems, vol. 24, no. 12, pp. 2027-2037, 2013. 


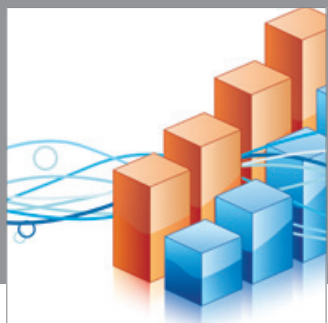

Advances in

Operations Research

mansans

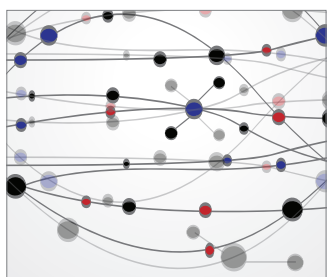

The Scientific World Journal
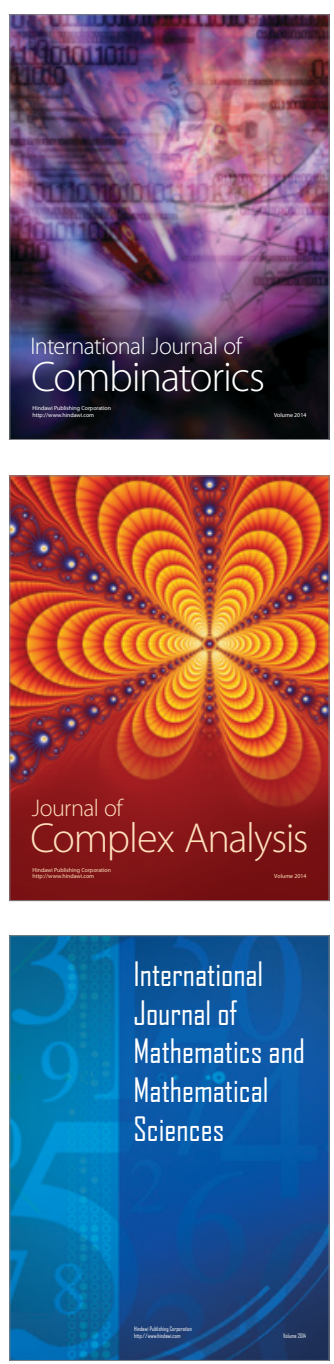
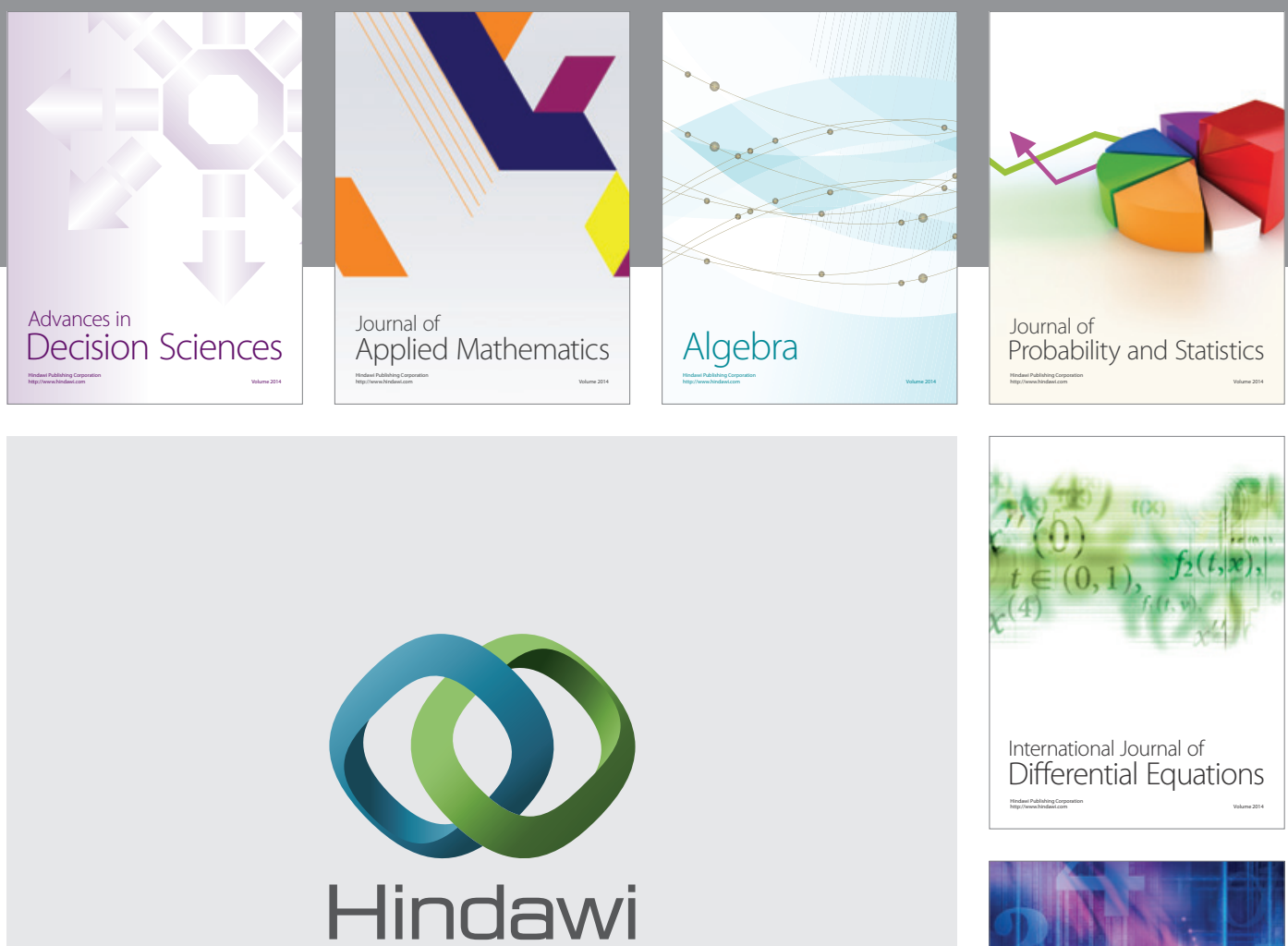

Submit your manuscripts at http://www.hindawi.com
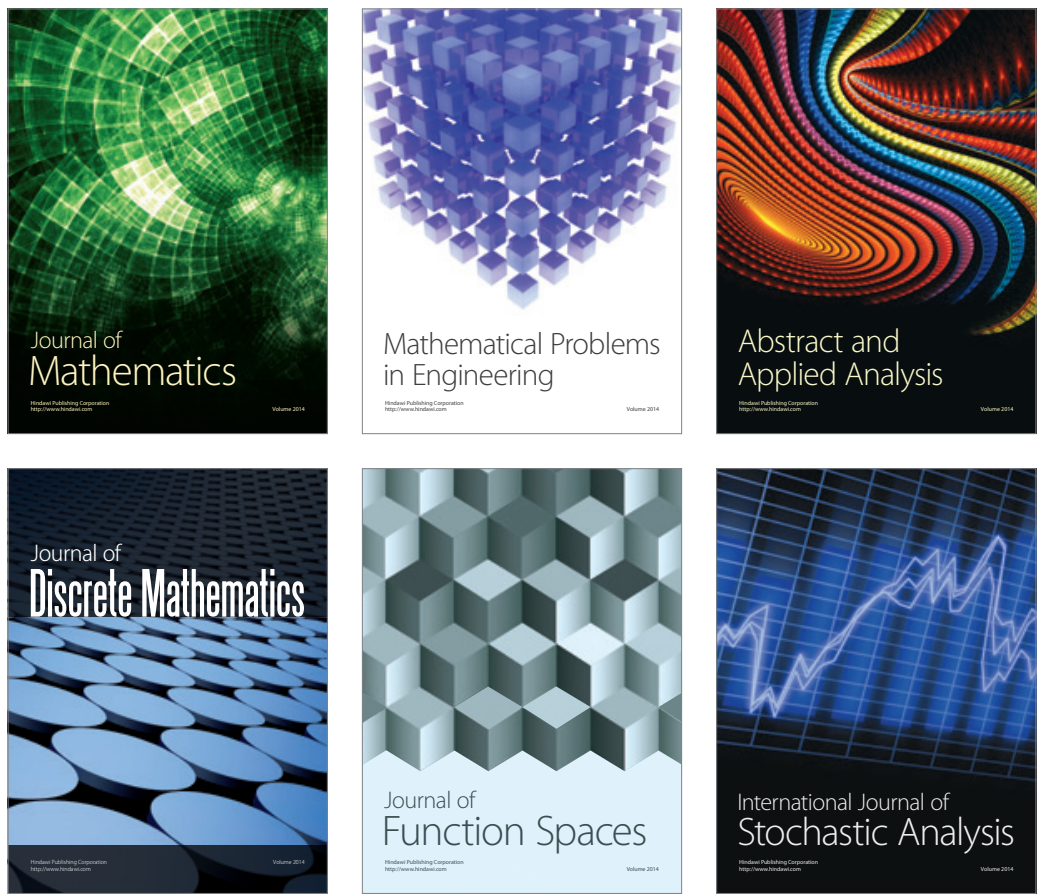

Journal of

Function Spaces

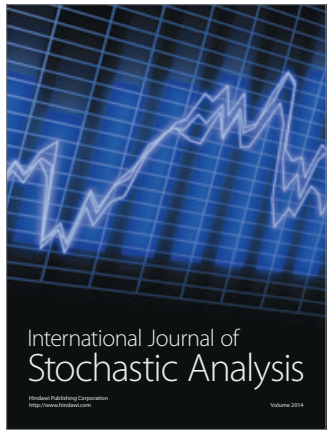

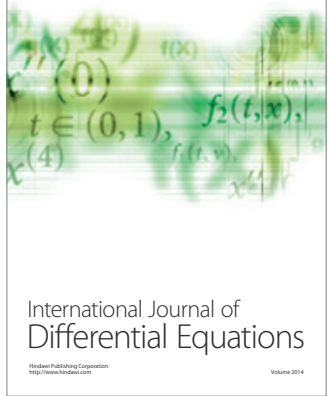
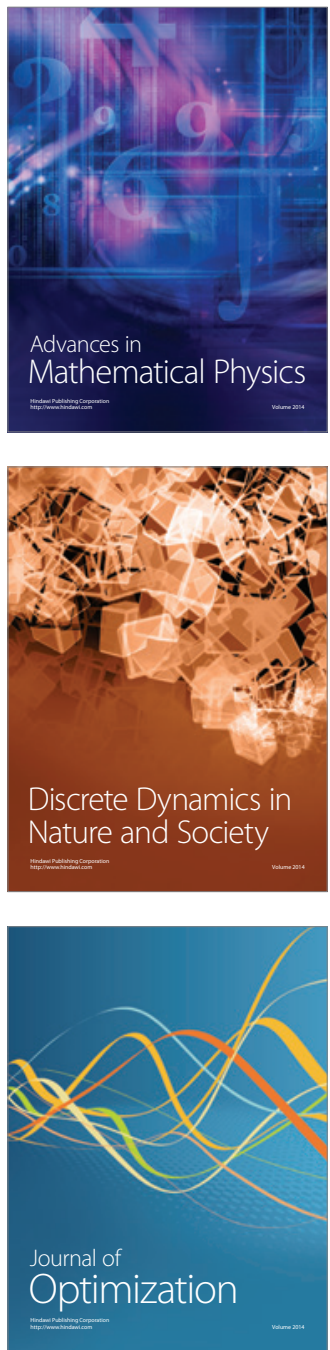